\title{
Transplacental Immunization of the Human Fetus to Tetanus by Immunization of the Mother
}

\author{
Thomas J. Gill ili, Charles F. Repetti, Leon A. Metlay, Bruce S. Rabin, \\ Floyd H. Taylor, Douglass S. Thompson, and Andrea L. Cortese, \\ Departments of Pathology, Community Medicine, and Obstetrics and \\ Gynecology, University of Pittsburgh School of Medicine and \\ Magee-Women's Hospital, Pittsburgh, Pennsylvania 15261
}

A B S T R A C T Experimental studies in rats showed that immunization of the pregnant female led to the transplacental immunization of her fetuses. The possibility that this also occurred in humans was explored by immunizing 42 pregnant women with tetanus toxoid $(2.5$ or $5 \mathrm{Lf})$ in the fifth and eighth months of pregnancy and comparing the immune responses of their offspring with the responses of the offspring of 25 unimmunized mothers. Only the offspring of the immunized mothers were sensitized to tetanus. IgM antitetanus antibodies were in their blood before immunization with diphtheria, pertussis, tetanus vaccine (DPT), they had a more rapid $(P<0.01)$ response to DPT immunization, and they were still highly sensitized $(P<0.01)$ to tetanus 13 mo after birth. In addition, pregnancy had no immunosuppressive effect $(P<0.05)$ on the responses of the mothers to tetanus toxoid. Thus, transplacental immunization occurs in humans; it enhances the response of the offspring to subsequent immunization, and it could be used to circumvent the necessity for immunization in early neonatal life.

\section{INTRODUCTION}

Experimental studies in inbred rats (1-5) showed that transplacental immunization of the fetus occurred: immunization of a pregnant animal with an insolubilized antigen permanently altered the immune response of its offspring $(1,2)$ due to the transplacental passage of antigen and its interaction with the developing immune system of the fetus (3), and the effect was ge-

Address reprint requests to Dr. Thomas J. Gill III, Department of Pathology, University of Pittsburgh, School of Medicine, Pittsburgh, PA 15261.

Received for publication 22 November 1982 and in revised form 28 April 1983. netically influenced (2-5). In this study we explore this phenomenon in humans. The basic significance of the study would lie in the demonstration that transplacental immunization, which was discovered in a species having a labyrinthine hemotrichorial placenta, occurs in another species, i.e., humans, which has a hemobichorial villous placenta. The clinical significance would lie in the possibility that the technique of transplacental immunization would provide a unique and safe approach to immunization in humans.

Tetanus toxoid (TT) ${ }^{1}$ was chosen as the antigen for this study because it is safe (6) and because there is extensive experience with tetanus immunization in humans (7-15). The experimental approach was to immunize females with TT at 5 and 8 mo of pregnancy and to assess the sensitization of their offspring to tetanus and their ability to respond to subsequent immunization with TT compared with the offspring of control (unimmunized) mothers. This stage of pregnancy was chosen for immunization because organogenesis and the major development of the fetus are finished, so any potential teratogenic effect of immunization would be improbable, and it is the most practical time from the clinical point of view. The doses of TT used were those ordinarily employed in immunizing adults ( 2.5 or $5 \mathrm{Lf}$ ) rather than the high doses (30-100 Lf) used in many field studies (9-15) or recommended for pregnant women exposed to tetanus (6) in order to avoid the possibility of inducing partial immunological tolerance in the offspring $(4,5)$. A second question to which this study could contribute is whether pregnant women can respond normally to immunization during pregnancy, which is still a point of some controversy (16).

\footnotetext{
'Abbreviations used in this paper: DPT, diphtheria, pertussis, tetanus vaccine; PHA, phytohemagglutinin; TT, tetanus toxoid.
} 


\section{METHODS}

Study population. The pregnant women were unscreened volunteers from the outpatient clinic at MageeWomen's Hospital. All of them had received three diphtheria, pertussis, tetanus vaccine (DPT) immunizations in childhood, and $12 \%(8 / 67)$ gave a history of one subsequent immunization with $\mathrm{T} T$ in childhood or in adolescence. The women entering the study were assigned in alternating sequence to the control (unimmunized) or immunized groups, and all of them were assayed for circulating antitetanus antibody. The immunized women were assigned randomly to receive a standard $(5 \mathrm{Lf})$ or half-standard $(2.5 \mathrm{Lf})$ dose of adult-type TT in order to test any potential effect of the amount of antigen used on the immune response. All of the TT used was from the same batch obtained from the Biological Laboratories, Massachusetts Department of Health. The mothers were vaccinated subcutaneously at 5 and $8 \mathrm{mo}$ of gestation and bled periodically (Fig. 1) thereafter for serum antibody and tetanus-induced lymphocyte transformation assays; the control mothers were assayed in parallel. The babies of both the control and the immunized mothers were assayed in the same way. Both groups of babies received the standard three doses of DPT: each dose contained 7.5 Lf diphtheria toxoid, 4 protective units pertussis vaccine, and $5 \mathrm{Lf}$ TT in $0.5 \mathrm{ml}$. The details of the experimental design are summarized in Fig. 1. All babies were normal by pediatric evaluation, both at birth and throughout the period of observation ( $3 \mathrm{yr}$ )

Assay methods. The antibody was measured by a radioimmunoassay that could detect $0.75 \mu \mathrm{g} / \mathrm{ml} \mathrm{IgG}$ and 0.4 $\mu \mathrm{g} / \mathrm{ml}$ IgM (17). The mixed lymphocyte reaction was performed as described previously (18). Briefly, heparinized blood from the mothers and babies was used to obtain lym- phocytes by Ficoll-Hypaque separation; the plasma layer was saved for autologous plasma. One batch of male AB plasma (BioBee Co., Boston, MA) was used in all studies. An appropriate dilution of lymphocytes, medium 199, and plasma (autologous or $\mathrm{AB}$ ) was prepared. Then $0.01 \mathrm{ml}$ of TT or phytohemagglutinin (PHA) (Burroughs-Wellcome Co., Research Triangle Park, NC) at the appropriate dilutions was added to each well in a 96 -well plate, and $0.2 \mathrm{ml}$ of the mixture of the cells, medium, and plasma was added. The mixture was incubated for $72 \mathrm{~h}$ and then pulsed with $2 \mu \mathrm{Ci} /$ well of $\left[{ }^{3} \mathrm{H}\right]$ thymidine. After a further 5-6 $\mathrm{h}$ of incubation, the cells were harvested and counted in a liquid scintillation counter. With tetanus stimulations, assays having $<100$ unstimulated (background) $\mathrm{cpm}$ were discarded, and with PHA stimulations, assays having $<5,000 \mathrm{cpm}$ were discarded. In either case, if the coefficient of variation of replicate counts was $>30 \%$, a replicate that was an obvious outlier ( $>2$ SD from the mean) was discarded. Only $3 \%$ of the data were discarded by these criteria. All of the cultures were done in duplicate in autologous plasma and in $A B$ plasma in order to look for potential immunosuppressive factors in the autologous plasma. The positive controls were the PHA-stimulated cultures (1:5 and 1:10 dilutions of PHA), and the negative controls were unstimulated cells. Three different amounts of TT $(0.5,1.0,2.9$ units) were used to stimulate the cultures in each of the two types of plasma.

TT-induced lymphocyte reactivity was scored as stimulated or unstimulated by a modification of the method of Harina et al. (19); the quantitative level of stimulation was not useful because of the intrinsic variability of the method (19). Briefly, a square-root transformation was performed on all control and stimulated counts. The difference $\bar{d}_{i}$ between the means of the stimulated and control counts was calculated, and the standard error of $\bar{d}_{i}, \operatorname{Var}_{w}\left(d_{i}\right)$, was calculated

\section{BABIES}

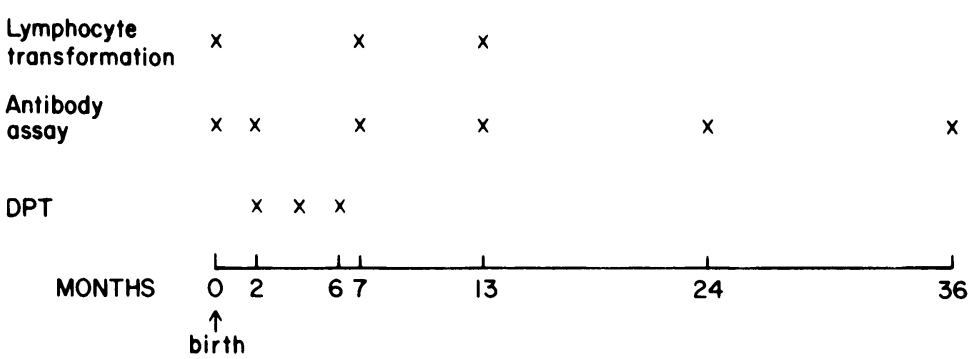

MOTHERS

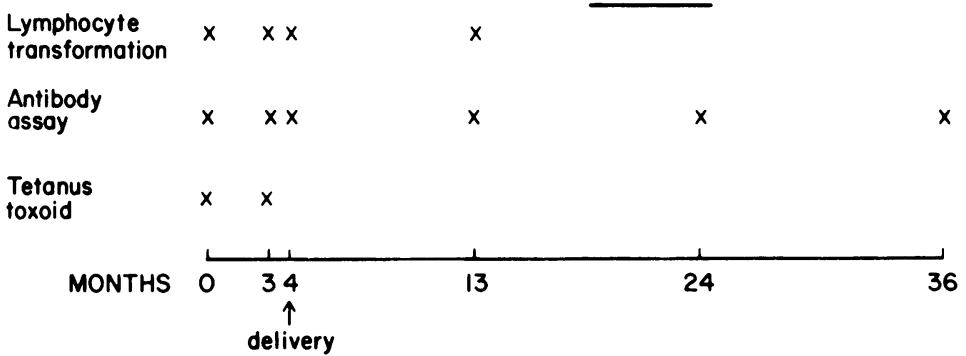

Figure 1 The experimental design of the maternal immunization study. The various procedures performed on the mothers and on the babies are indicated on the appropriate time axis. The TT was given to the mothers after the bleedings at 0 and 3 mo, and the first DPT immunization was given to the babies after the bleeding at 2 mo. 
from the pooled variances of control and stimulated counts and the estimated covariances of control and stimulated counts. The variances were pooled separately for each group of subjects and for each bleeding. An individual group of replicates was scored as stimulated if $\bar{d}_{i} \geqslant t_{n-1} \operatorname{Var}_{w}\left(d_{i}\right)$, where $t_{n-1}$ is the critical value of the $t$-statistic for $P=0.05$ and $n-1$ degrees of freedom, and $n$ is the number of replicates pooled in a group. There were no significant differences in the responses elicited by the three dilutions of TT or by the two dilutions of PHA, so the responses were treated as replicates.

Statistical treatment of data. The G-statistic (likelihood ratio) was used in tests of independence. The McNemar test of change was used in comparisons among the lymphocyte transformation reactions when two tests in the same individual were compared (20).

Three types of analyses were done to evaluate the data before using them to answer the experimental questions. First, the effect of the antibody status of the mothers before immunization, i.e., whether they had detectable IgG or IgM antitetanus antibody, was examined to determine its effect on the antibody and lymphocyte transformation responses of their offspring. Second, lymphocyte stimulations were compared in autologous plasma and in $\mathrm{AB}$ plasma in order to test for the presence of any immunosuppressive factors in the autologous plasma. Third, the effects of immunizing with a standard or half-standard dose of TT on the antibody and lymphocyte transformation responses of the mothers and the babies were examined.

If none of the variables affected the results, the data were pooled and used to answer two questions. First, are the babies of immunized mothers sensitized to tetanus by transplacental immunization? The criteria for sensitization were: the presence of IgM antitetanus antibody in the cord blood and in the serum before the first DPT immunization; the level of responsiveness to DPT immunization as measured by anti- body and lymphocyte transformation assays, and the level of lymphocyte responsiveness 1 yr after birth. Second, can the pregnant female respond to immunization with TT as measured by antibody and lymphocyte transformation responses?

\section{RESULTS}

The demographic data for the mothers in the study and for their offspring at birth are summarized in $\mathrm{Ta}$ ble I. There were no significant differences among the three groups in the study. Seven women who were tetanus-negative at the beginning of the study were not immunized, and their antibody levels were followed. Six of them subsequently developed measureable amounts of antitetanus antibody, so they had been sensitized to tetanus but their antibody levels at the beginning of the study were undetectable. Similar evidence for tetanus sensitization in women who were ostensibly tetanus-negative when the study began was obtained from lymphocyte transformation studies in autologous plasma and in $\mathrm{AB}$ plasma, which showed no differences in the responses to stimulation by TT between mothers who were tetanus negative or tetanus positive when entering the study (Table II).

To test whether the presence of autologous plasma or of AB plasma influenced the response of lymphocytes to stimulation by TT, assays were performed in parallel in both plasmas and compared by the McNemar test of change: 252 assays were performed with

TABLE I

Demographic Data on the Mothers and Babies ${ }^{\bullet}$

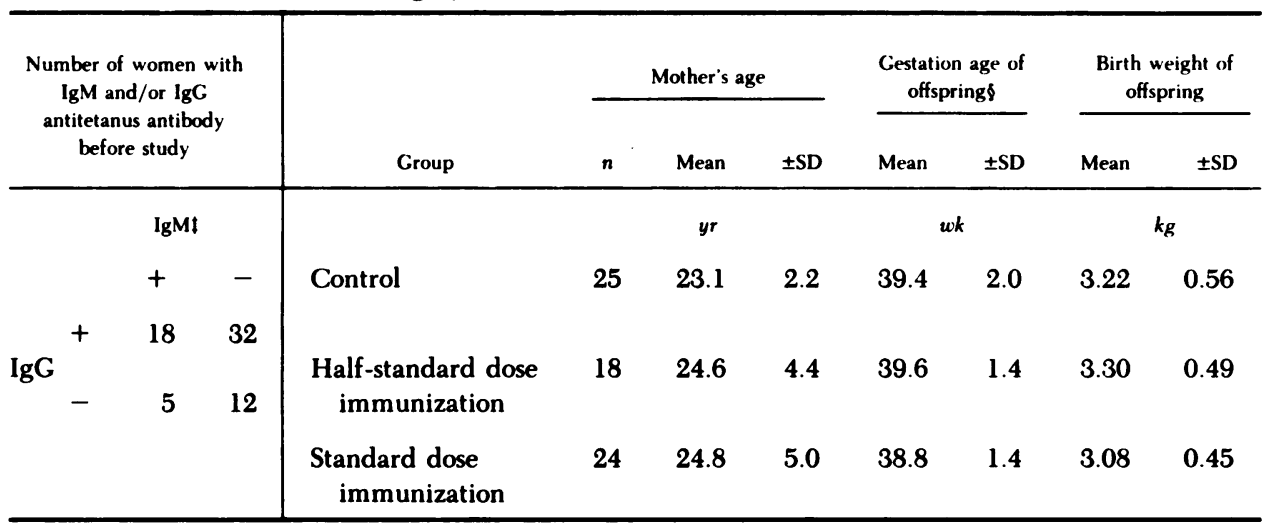

\footnotetext{
- Age of females, gestational age, and birth weight differences were assessed by the $F$-ratio. All other variables were assessed by the $\boldsymbol{G}$-statistic with 0.1 added to each cell to compensate for any values of 0 . No significant differences in sex or feeding pattern (breast or bottle) among offspring in the different classes $(P>0.2)$. No significant differences in gravidity, parity, or race (black or white) among the mothers in the three groups $(P>0.1)$.

$\downarrow+$, present; - , absent.

$\$$ No significant differences among groups $(P>0.6)$.
} 
TABLE II

Effect of the Initial Tetanus Status of the Mothers on the Stimulation of Their Lymphocytes in AB Plasma and in Autologous Plasma

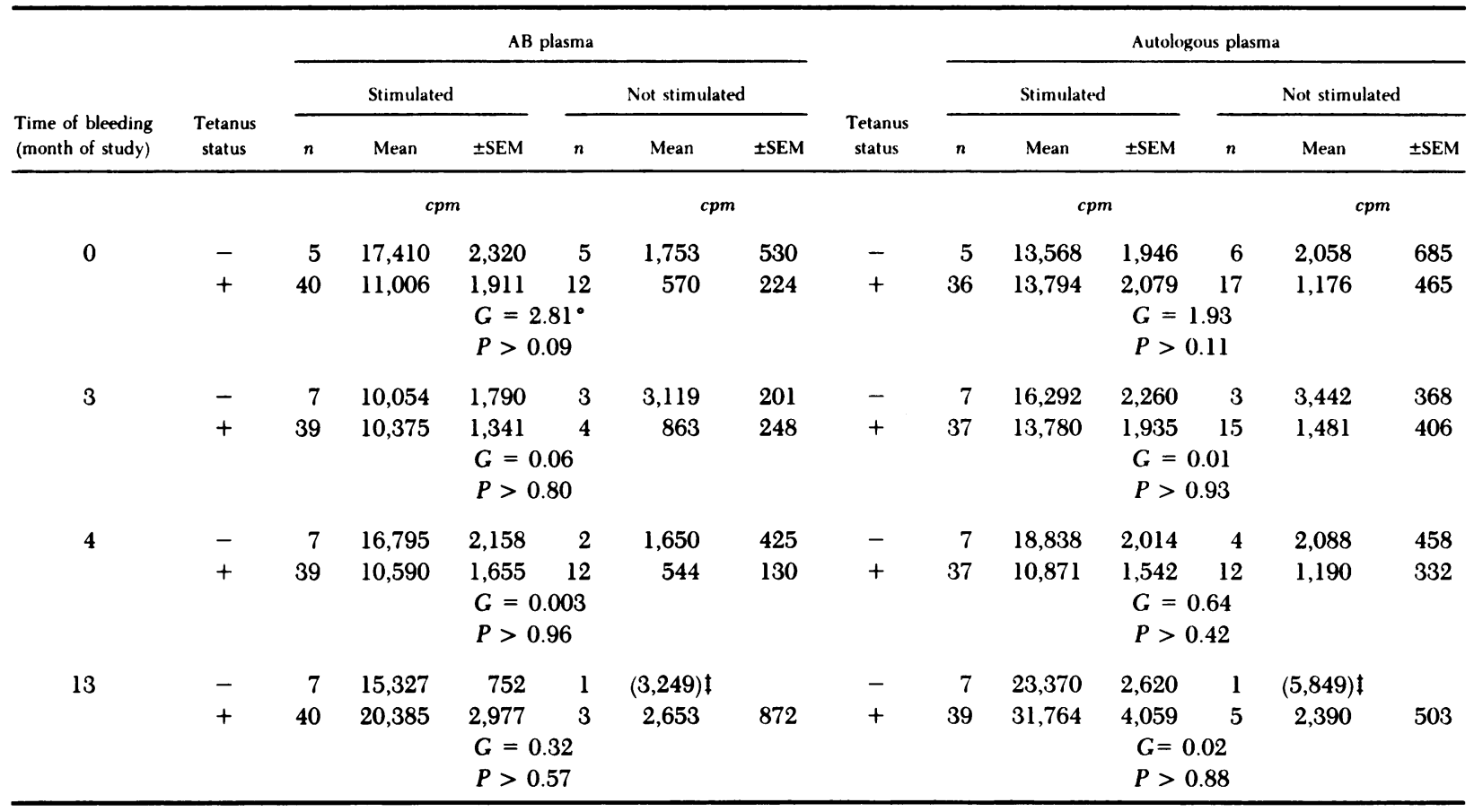

- The $G$-statistic was used for the comparisons between the number of mothers whose lymphocytes were stimulated and those whose lymphocytes were not stimulated. The mothers were further categorized as being either tetatnus negative or tetanus positive at the beginning of the study. The counts per minute are for stimulation with 0.5 units of TT. The levels of incorporation with the other two concentration of TT used (1.0 and 2.0 units) were the same.

† One value only.

lymphocytes from control and immunized mothers; 112 assays, from nonpregnant women, and 212 assays, from babies born to control and immunized mothers. None showed any differences in lymphocyte stimulation in the two plasmas; therefore, the data were pooled and used as replicate assays. There were no differences in the lymphocyte transformation responses to TT between women immunized with a standard or half-standard dose of TT, using the $G$-statistic for the comparisons, in 18/19 cases. There were also no differences in the responses of the babies born to mothers immunized with a standard or half-standard dose of TT (Fig. 2). Finally, there was no significant effect of the dose of TT used for stimulation on the lymphocyte responses of the mothers or of their babies.

The babies of mothers immunized with TT had IgM antitetanus antibodies in their cord blood, whereas the babies of control mothers did not, and the prevalence and the amount of the antibodies were higher in the babies of mothers who received a standard dose of TT (Table III, Fig. 2). The tetanus-induced lymphocyte stimulation studies also supported the finding of trans- placental immunization of the offspring. The major piece of evidence comes from a comparison of the number of babies from control and immunized mothers whose lymphocytes were stimulated or not stimulated by TT at various times after birth (Table IV). The babies of immunized mothers responded earlier (by 7 mo of age) to DPT immunization than did the babies of control mothers. More of the babies from immunized mothers had lymphocytes sensitized to TT at 13 mo of age than had sensitized lymphocytes at 7 mo of age $(P<0.01)$. By contrast, there was no significant difference in the number of babies with sensitized lymphocytes at 7 and 13 mo among the offspring of control mothers. The level of lymphocyte stimulation, or lack thereof, as measured by thymidine incorporation, was the same in the lymphocytes of the babies from control or immunized mothers (Table V). A comparison of the percentage of babies with stimulated lymphocytes also shows that more of the babies of immunized mothers had sensitized lymphocytes at 7 and 13 mo than did the babies of control mothers (Table V). There was no significant difference in the 

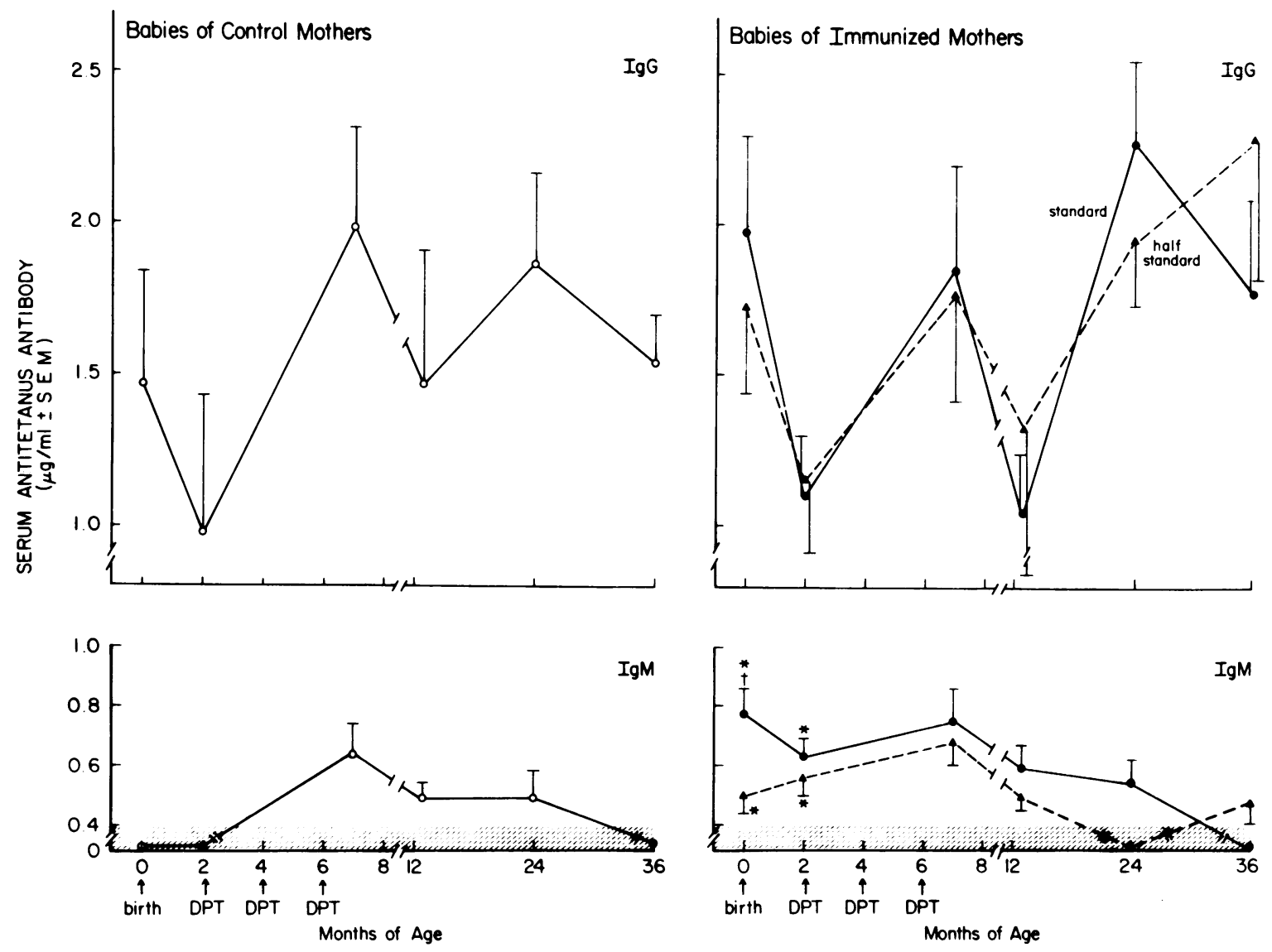

Figure 2 The presence of antitetanus antibodies in the babies of control (unimmunized) and immunized mothers and the babies' responses to immunization with DPT. Each point represents data from 12 to 23 babies, and the shaded area delineates the sensitivity of the assay method. Babies of immunized mothers had IgM in their cord blood and in their blood before the first immunization with DPT, whereas babies of control mothers did not. In addition, the level of IgM was higher in the cord blood of babies whose mothers received the standard dose of TT. The asterisks indicate a significant difference $(P<0.05)$ between the babies of control mothers and those of immunized mothers, and the daggers indicate a significant difference $(P<0.05)$ between the babies whose mothers received the standard dose of TT and those whose mothers received the half-standard dose of TT. The solid line is the response of babies whose mothers received a standard dose of $\mathrm{TT}$, and the broken line is the response of babies whose mothers received a half-standard dose of TT. There was no difference between the IgG antitetanus antibody levels in the babies of control and immunized mothers or between babies of mothers receiving a standard or a half-standard dose of $\mathrm{TT}$. The significance of the differences was assessed by the $G$-statistic.

initial levels of IgG antitetanus antibody in the babies of the control and immunized mothers, as would be expected because of the transplacental passage of IgG antibodies from the mothers, or in the IgG response to DPT immunization (Fig. 2).

The immunized mothers responded quite well to immunization with TT (Fig. 3). The increase in IgG antitetanus antibody production was significant both in the group receiving the standard dose of TT $(P$ $<0.05)$ and in the group receiving the half-standard dose $(P<0.005)$, and it exceeded the level of IgG in the control mothers $(P<0.05)$. The difference in IgM production between the immunized and control groups was not statistically significant, although IgM levels were consistently higher in the immunized mothers. No untoward effects of immunization with TT were observed in the mothers or their offspring.

\section{DISCUSSION}

Three lines of evidence show that transplacental immunization of the human fetus occurs following im- 
TABLE III

Prevalence of IgM Antitetanus Antibody in the Offspring of Control and Immunized Mothers Before Their First DPT Immunization

\begin{tabular}{|c|c|c|c|c|c|c|}
\hline \multirow{4}{*}{$\begin{array}{l}\text { Presence of antibody } \\
\text { in the mother before } \\
\text { immunization }\end{array}$} & \multicolumn{6}{|c|}{ Offspring with IgM from mothers with different vaccination status } \\
\hline & & & \multicolumn{4}{|c|}{ Immunized mothers } \\
\hline & \multicolumn{2}{|c|}{ Control mothers } & \multicolumn{2}{|c|}{ Half-standard dose } & \multicolumn{2}{|c|}{ Standard dose } \\
\hline & $n$ & Percentage & $n$ & Percentage & $n$ & Percentage \\
\hline 0 & $0 / 3$ & 0 & $0 / 5$ & $\mathbf{0}$ & $3 / 4$ & 75 \\
\hline \multirow[t]{2}{*}{+} & $0 / 21$ & $\mathbf{0}$ & $8 / 14$ & 57 & $13 / 20$ & 65 \\
\hline & $0 / 24$ & 0 & $8 / 19$ & 42 & $16 / 24$ & 67 \\
\hline
\end{tabular}

munization of mothers during the fifth and eighth months of pregnancy. First, there was IgM antitetanus antibody in the babies' blood before the first DPT immunization, whereas it was not present in the blood of babies from control (unimmunized) mothers (Table IV, Fig. 3). Second, there was a higher lymphocyte transformation response $(P<0.01)$ to DPT immunization in the babies of immunized mothers (Table V). Third, the lymphocytes from babies of the immunized mothers were still highly sensitized to TT 13 mo after birth, whereas those from babies of the control (unimmunized) mothers were not $(P<0.01)$ (Table V). These studies in humans reproduce the original experimental observations in rats upon which the protocol was based (1-5). They show that active immunization of the pregnant female can sensitize her fetus and that the difference in placental structure in the two species does not affect the transplacental passage of antigen. Studies on pregnant women infected with influenza $(21)$, leprosy $(22,23)$, rubella $(24,25)$, hepatitis $(26,27)$, mumps (28), Escherichia coli (29) or Salmonella typhosa (30) showed that their offspring were sensitized to the microbial antigens, but the possibility of direct infection of the fetus or of placental damage leading to the sensitization cannot be eliminated in this setting.

It is highly unlikely that the observations reported here could be due to the transplacental passage of antibodies and sensitized cells from the mother. First, the concentration of IgM antibody is approximately the same in the immunized mothers and in their babies (Figs. 2 and 3). In order for this to occur by transpla-

TABLE IV

Comparison of the Number of Babies from Control and Immunized Mothers Whose Lymphocytes Are Stimulated or Not Stimulated by TT at Different Times After Birth

\begin{tabular}{|c|c|c|c|c|c|c|c|c|c|}
\hline \multirow{3}{*}{$\begin{array}{c}\begin{array}{c}\text { Maternal } \\
\text { group }\end{array} \\
\text { Control }\end{array}$} & \multirow[b]{2}{*}{${ }_{0} \mathrm{mo}$} & \multirow{2}{*}{\multicolumn{2}{|c|}{7 mo }} & & & & & & \\
\hline & & & & $0 \Gamma_{\mathrm{mo}}$ & \multicolumn{2}{|c|}{$13 \mathrm{mo}$} & $\Gamma_{7 \mathrm{mo}}$ & \multicolumn{2}{|c|}{$13 \mathrm{mo}$} \\
\hline & & + & - & & + & - & & + & - \\
\hline & + & 2 & $\mathbf{0}$ & + & 2 & 1 & + & 5 & 3 \\
\hline & - & 4 & 2 & - & 7 & 1 & - & 2 & 1 \\
\hline & $\mathrm{X}^{2}=$ & & & $\mathrm{X}^{2}=$ & & & & $\mathrm{X}^{2}$ & \\
\hline & $P>$ & & & $P<$ & & & & $P=$ & \\
\hline \multirow[t]{5}{*}{ Immunized } & & + & - & & + & - & & + & - \\
\hline & + & 2 & $\mathbf{l}$ & + & 4 & 0 & + & 12 & 1 \\
\hline & - & 11 & 11 & - & 18 & 3 & - & 10 & 1 \\
\hline & \multicolumn{3}{|c|}{$\mathrm{X}^{2}=8.20$} & \multicolumn{4}{|c|}{$X^{2}=1780$} & \multicolumn{2}{|c|}{$X^{2}=7.23$} \\
\hline & \multicolumn{3}{|c|}{$P<0.01$} & \multicolumn{3}{|c|}{$P<10^{-4}$} & & \multicolumn{2}{|c|}{$P<0.01$} \\
\hline
\end{tabular}

- McNemar test of change was used for the comparisons. The $(+)$ indicates stimulation, and the $(-)$, lack of stimulation.

$10 \mathrm{mo}$ is the cord blood, and $7 \mathrm{mo}$ is $1 \mathrm{mo}$ after the last DPT immunization. 
TABLE V

Levels of Thymidine Incorporation into the Lymphocytes of Babies from Control and Immunized Mothers.

\begin{tabular}{|c|c|c|c|c|c|c|c|c|c|c|}
\hline \multirow{3}{*}{$\begin{array}{c}\text { Maternal } \\
\text { group }\end{array}$} & \multirow[b]{3}{*}{ Babies' lymphocyte response } & \multicolumn{9}{|c|}{ Thymidine incorporation by babies' lymphocytes at different ages } \\
\hline & & \multicolumn{3}{|c|}{0 mo (cord blood) } & \multicolumn{3}{|c|}{$7 \mathrm{mo}$} & \multicolumn{3}{|c|}{$13 \mathrm{mo}$} \\
\hline & & $n$ & Mean & \pm SEM & $n$ & Mean & \pm SEM & $n$ & Mean & \pm SEM \\
\hline & & & \multicolumn{2}{|c|}{$c p m$} & \multicolumn{3}{|c|}{$c p m$} & & \multicolumn{2}{|c|}{ cpm } \\
\hline \multirow[t]{3}{*}{ Control } & Stimulated & 6 & 7,334 & 1,247 & 2 & 17,407 & 2,502 & 2 & 19,741 & 2,825 \\
\hline & Not stimulated & 14 & 931 & 542 & 6 & 2,420 & 752 & 9 & 3,114 & 523 \\
\hline & Percentage stimulated & & \multicolumn{2}{|c|}{$30 \%$} & \multicolumn{3}{|c|}{$25 \%$} & & \multicolumn{2}{|c|}{$18 \%$} \\
\hline \multirow[t]{3}{*}{ Immunized } & Stimulated & 14 & 8,735 & 1,284 & 13 & 10,998 & 1,910 & 22 & 22,584 & 1,970 \\
\hline & Not stimulated & 30 & 1,194 & 422 & 12 & 1,303 & 430 & 3 & 2,706 & 686 \\
\hline & Percentage stimulated & & \multicolumn{2}{|c|}{$32 \%$} & \multicolumn{4}{|c|}{$52 \%$} & \multicolumn{2}{|c|}{$88 \%$} \\
\hline
\end{tabular}

The data for lymphocytes that were either stimulated or not stimulated by TT are given.

cental transport from the mother, there would have to be free diffusion of IgM across the placenta or selective transport of $\operatorname{IgM}$ antitetanus antibodies; there is substantial evidence that neither occurs (31). Even if the rate of transport of IgM across the placenta were proportional to the inverse square root of its molecular weight, as occurs with smaller proteins (31), the change in the fetal/maternal plasma concentration ratio of IgM would be of the order of $10^{-3} / \mathrm{d}$. This low ratio and the relatively short half-life of $\operatorname{IgM}(5 \mathrm{~d})$ would allow only a few nanograms at most of maternal IgM antibody to be present in the fetus. Experimental support for this conclusion comes from the observation that only the babies of immunized mothers had IgM antitetanus antibodies in their sera at birth, although both the control and immunized mothers had comparable concentrations of IgM antibodies in their sera at the time of delivery (Fig. 3). Second, there is no clear evidence for the passage of maternal lymphocytes into the normal fetus during pregnancy, although it has been reported that passage at delivery occurs in $0-30 \%$ of cases (reviewed in references 16 and 33 ). Even if it did occur, colonization of the fetus by tetanus-sensitized lymphocytes from the mothers could not explain the findings in the babies. The same percentage of babies from control and immunized mothers had sensitized lymphocytes at birth, but the number of babies from immunized mothers who developed sensitized lymphocytes after birth increased rapidly, whereas the number of babies from control mothers with sensitized lymphocytes decreased after birth (Table V). Such a change would not occur if the lymphocytes in both groups of babies have originated in the mothers. Evidence from demonstrated maternal colonization in other circumstances also militates against maternal colonization of the babies in this study. In patients with severe combined immunodeficiency disease, where there should be relatively little resistance to maternal lymphocytic colonization, only 4/16 patients showed engraftment (32), and the engrafted lymphocytes did not respond to stimulation with mitogens, antigens, or allogeneic cells $(32,33)$. Following intrauterine transfusion of male infants with maternal lymphocytes, only 5/46 infants had maternal lymphocytes present at birth, and they persisted to 1 yr of age (34). Following postnatal exchange transfusion, $34 / 82$ babies had detectable donor lymphocytes at birth, but only $1 / 82$ had donor lymphocytes detectable at 4 mo of age (34). Thus, the persistance of maternal lymphocytes in the babies could not explain the presence of tetanus-sensitized cells in $88 \%$ of the offspring of immunized mothers at 13 mo of age (Table V). From studies in animals in which transplacental passage of maternal lymphocytes occurred, one would expect to see a graft-vs.-host reaction (35) or immunosuppression (36) in the offspring, and, indeed, at least several such cases have been reported in humans $(32,37-39)$. Neither phenomenon was observed in any of the babies in our study. Finally, stimulation of the lymphocytes cannot be due to a mitogenic effect of TT, at least to any significant degree. If TT were acting as a mitogen, the percentage of babies from control mothers whose lymphocytes were stimulated would not decrease with the age of the babies nor would there be the marked differences between the percentages of babies from control and immunized mothers whose lymphocytes were stimulated by TT (Table V).

The genetic control of the responsiveness to TT $(40$, 41) could not be examined in this study because the mother and father would have to be either homozygous responders or homozygous nonresponders in order to 

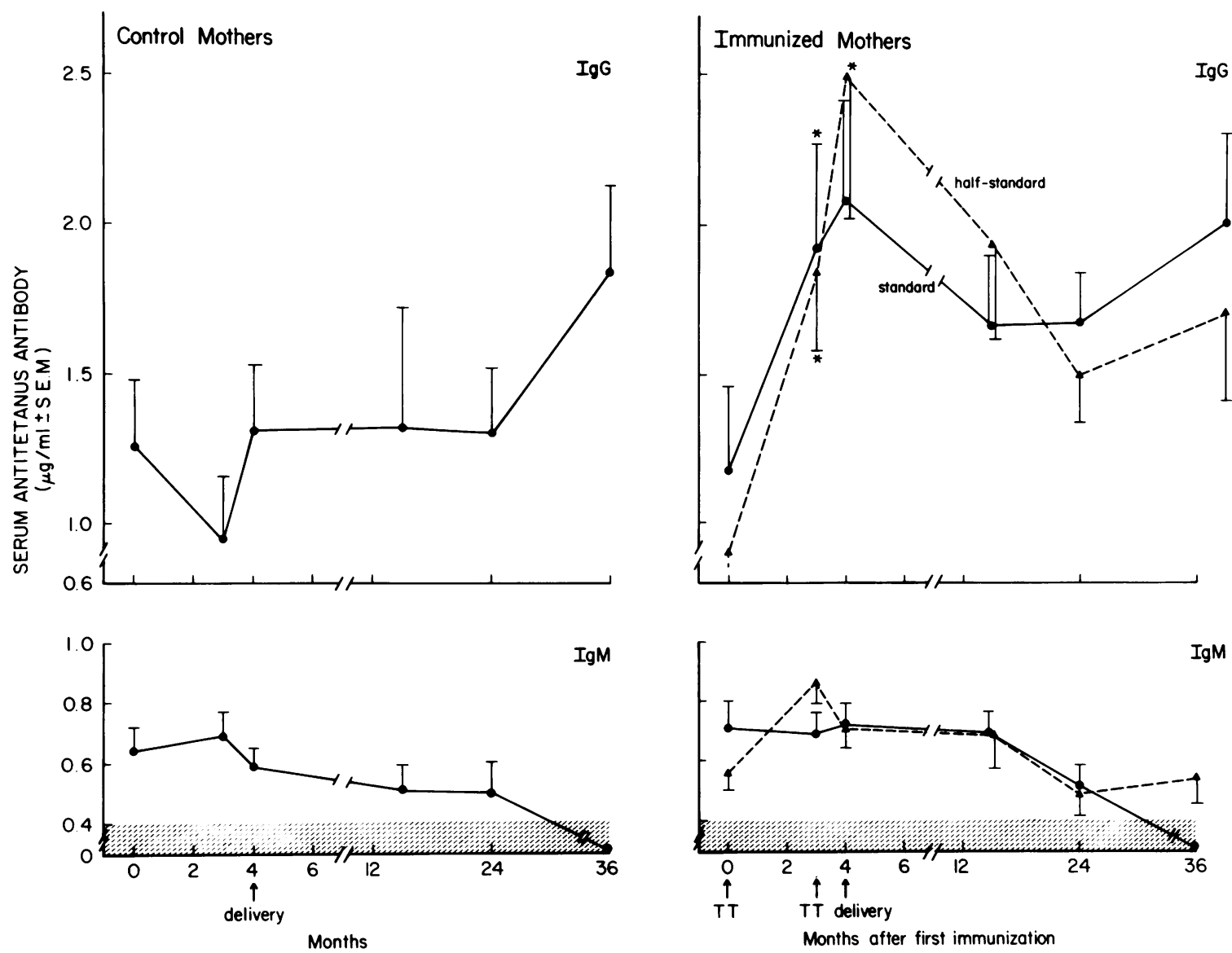

FIGURE 3 The production of IgG and IgM antitetanus antibodies in control (unimmunized) and immunized mothers. Each point represents data from 12 to 24 mothers, and the shaded area delineates the sensitivity of the assay method. The mothers receiving TT responded to immunization $(P<0.05-<0.005)$, and there was no significant difference between the responses of those receiving a standard $(5 \mathrm{Lf})$ and a half-standard $(2.5 \mathrm{Lf})$ dose of TT. The asterisks indicate a significant difference $(P<0.05)$ between the control and immunized mothers in IgG antitetanus antibody production. Although there was no statistically significant difference in IgM antibody production between the control and immunized mothers, the level of IgM antibody in the immunized mothers remained higher than in the control mothers. The significance of the differences was assessed by the $G$-statistic.

detect a genetic influence on the immune response of the offspring: a heterozygous offspring would have an intermediate response that would be difficult to classify. It is fortunate that matings between homozygous responders are probably uncommon in human populations, because the probability of maternal immunization's causing decreased responsiveness to tetanus in the offspring due to the induction of partial tolerance $(4,5)$ is remote.

Two observations indicate that there is no functional evidence for an immunosuppressive effect during pregnancy. First, the pregnant females responded well to immunization with TT (Fig. 3); a similar finding was noted following immunization with influenza vac- cine $(42,43)$. Second, the lymphocyte responses were not different in autologous plasma and in $A B$ plasma. Thus, there do not appear to be any physiologically active, immunosuppressive plasma factors in the pregnant female, at least during the fifth and eighth months of gestation and at delivery. This observation contributes to the body of evidence supporting the hypothesis that the acceptance of the fetal homograft is not due to immunosuppression in the mother, although arguments can still be made for local (uterine) effects of immunosuppressive substances that may not be reflected systematically.

The technique of transplacental immunization may provide a unique and safe approach to immunization 
in humans, and it may be especially useful in those populations where neonatal care is not readily available. It has substantial potential for clinical use to protect the offspring during the neonatal period and to enhance its response to subsequent immunization or to exogenous infection. It also circumvents the necessity for immunization in the early postnatal period, when the levels of maternal IgG antibody in the newborn are still high and could interfere with the efficacy of the immunization procedures. The enhanced immunity due to transplacental immunization, at least as judged by the persistence of sensitized lymphocytes (Tables IV and V), lasts longer than $1 \mathrm{yr}$, but the total duration of its protective effect can only be judged by long-term population studies. Transplacental immunization may also be useful with other clinically important vaccines, such as those against the Streptococcus and the Meningococcus, since they are inert polysaccharides which can pass through the placenta (44). This approach might even be useful with vaccines against diseases that occur in later life, such as gonococcal infection, if the transplacentally induced immunity lasts long enough and if effective vaccines are available.

\section{ACKNOWLEDGMENTS}

We thank Mr. Leo Levine of the Biological Laboratories, Massachusetts Department of Health, for providing the tetanus toxoid; Dr. Gurmukh Singh, Dr. Jerome Sachs, and Dr. Paul M. Taylor for helpful discussions; and Ms. Katherine Rumin, Ms. Mary Ann Kofke, Mr. Bruce Hyde, Ms. Dorothy Crowell, and Ms. Megan B. Taylor for technical assistance.

This study was supported by National Institutes of Health grant HD 09880.

\section{REFERENCES}

1. Gill, T. J., III, and H. W. Kunz. 1971. Enhanced antibody response in the offspring of immunized rats. J. Immunol. 106:274-275.

2. Gill, T. J., III, H. W. Kunz, and C. F. Bernard. 1971. Maternal-fetal interactions and immunological memory. Science (Wash. DC). 172:1346-1347.

3. Gill, T. J., III, H. W. Kunz, and B. K. Davis. 1977. Effect of maternal immunization on the antibody response of low-responder rats. Int. Arch. Allergy Appl. Immunol. 53:366-375.

4. Davis, B. K., and T. J. Gill III. 1975. Decreased antibody response in the offspring of immunized high-responder rats. J. Immunol. 115:1166-1168.

5. Cramer, D. V., T. J. Gill III, and G. Knauer. 1978. The influence of maternal immunization on the antibody response of the offspring in genetically high-responding rats. Am. J. Pathol. 90:317-323.

6. Report of the Committee on Infectious Diseases. 1977. Am. Acad. Pediatr. 14:283-284.

7. Ipsen, J., Jr. 1954. Immunization of adults against diphtheria and tetanus. N. Engl. J. Med. 251:459-466.

8. Gottlieb, S., F. X. McLaughlin, L. Levine, W. C. Lathan, and G. Edsall. 1964. Long-term immunity to tetanus- a statistical evaluation and its clinical implications. Am. J. Public Health. 54:961-971.

9. LaForce, F. M., L. S. Young, and J. V. Bennett. 1969. Tetanus in the United States (1965-1966). Epidemiologic and clinical features. N. Engl. J. Med. 280:569574

10. Newell, K. W., D. R. Leblanc, L. Levine, H. Christiansen, M. H. Montouri, and N. Ramirez. 1971. The serological assessment of a tetanus toxoid field trial. Bull. W. H. O. 45:773-785.

11. Relyveld, E. H., R. Labusquiere, C. Gateff, F. LeBourthe, P. Ravisse, G. Lemarinier, and L. Chambon. 1972. Antitetanus vaccination and neonatal protection in developing countries. Prog. Immunobiol. Stand. 5:517-527.

12. Stanfield, J. P., D. Gall, and P. M. Bracken. 1973. Singledose antenatal tetanus immunization. Lancet. I:215219.

13. Garnier, M. J., F. N. Marshall, K. J. Davison, and F. J. Lepreau, Jr. 1975. Tetanus in Haiti. Lancet. I:383-386.

14. Suri, J. C., H. Dhillon, and H. S. Grewal. 1964. Active immunization of women in pregnancy for prevention of neonatal tetanus. Bull. W. H.O. 31:349-357.

15. Schofield, F. D., V. M. Tucker, and G. R. Westbrook 1961. Neonatal tetanus in New Guinea. Effect of active immunization in pregnancy. Br. Med. J. 2:785-789.

16. Gill, T. J., III, and C. F. Repetti. 1979. Immunologic and genetic factors influencing reproduction. Am. J. Pathol. 95:465-570.

17. Repetti, C. F., and T. J. Gill III. 1980. Radioimmunoassay of IgM and IgG antitetanus toxoid antibody. $J$. Immunol. Methods. 37:153-163.

18. Gill, T. J., III, B. S. Rabin, B. M. Harina, and F. H. Taylor. 1979. Relationship between the responsiveness of maternal and fetal lymphocytes to phytohaemagglutinin and to microbial antigens. J. Immunogenet. (Oxf.). 6:197-214.

19. Harina, B. M., T. J. Gill III, B. S. Rabin, and F. H. Taylor. 1978. A statistical analysis of human lymphocyte transformation data. J. Immunogenet. (Oxf.). 6:185196.

20. Sokal, R. R., and F. J. Rohlf. 1981. Biometry. W. H. Freeman \& Company Publishers, San Francisco. Second ed. 691-747; 765-774.

21. Ruben, F. L., and D. S. Thompson. 1981. Cord blood lymphocyte in vitro responses to influenza $A$ antigens after an epidemic of influenza $\mathrm{A} /$ Port Chalmers/73 $\left(\mathrm{H}_{3} \mathrm{~N}_{2}\right)$. Am. J. Obstet. Gynecol. 141:433-437.

22. Melsom, R., M. E. Duncan, M. Harboe, and G. Bjune. 1980. Antibodies against Mycobacteriun leprae antigen 7 from birth to 18 months of age: an indicator of intrauterine infection in leprosy. Clin. Exp. Immunol. 42:107113.

23. Melsom, R., M. E. Duncan, and G. Bjune. 1980. Immunoglobulin concentration in mothers with leprosy and in healthy controls and their babies at the time of birth. Lepr. Rev. 51:19-28.

24. Ruben, F. L., A. Winkelstein, and R. E. Sabbagha. 1975 In utero sensitization with influenza virus in man. Proc. Soc. Exp. Biol. Med. 149:881-883.

25. Cradock-Watson, J. E., M. K. S. Ridehalgh, M. J. Anderson, J. R. Pattison, and H. O. Kangro. 1980. Fetal infection resulting from maternal rubella after the first trimester of pregnancy. J. Hyg. (Camb.) 85:381-391.

26. Schweitzer, I. L., A. Wing, C. McPeak, and R. L. Spears. 1972. Hepatitis and hepatitis-associated antigen in 56 
mother-infant pairs. JAMA (J. Am. Med. Assoc.). 220:1092-1095.

27. Papaevangelou, G., J. Hoofnagle, and J. Kremastinou. 1974. Transplacental trasmission of hepatitis $B$ virus by symptom-free chronic carrier mothers. Lancet. II:746748.

28. Aase, J. M., G. R. Noren, D. V. Reddy, and J. W. St Geme, Jr. 1972. Mumps-virus infection in pregnant women and the immunologic response of their offspring. N. Engl. J. Med. 286:1379-1382.

29. Brody, J. I., F. A. Oski, and E. E. Wallach. 1968. Neonatal lymphocyte reactivity as an indicator of intrauterine bacterial contact. Lancet. I:1396-1398.

30. Bellanti, J. A., and A. L. Jackson. 1967. Characterization of the serum immunoglobulins to the somatic antigen of $S$. typhosa in an infant following intrauterine immunization. J. Pediatr. 71:783-789.

31. Gitlin, D. 1974. Protein transport across the placenta and protein turnover between amniotic fluid, maternal, and fetal circulations. In The Placenta. K. S. Moghissi and E. S. E. Hafez, editors. Charles C Thomas, Publisher, Springfield, IL. 151-191.

32. Pollack, M. S., D. Kirkpatrick, N. Kapoor, B. Dupont, and R. J. O'Reilly. 1982. Identification by HLA typing of intrauterine-derived maternal $\mathrm{T}$ cells in four patients with severe combined immunodeficiency. N. Engl. J. Med. 307:662-666.

33. Pollack, M. S., N. Kapoor, M. Sorell, S. J. Kim, F. T. Christiansen, D. M. Silver, B. Dupont, and R. J. O'Reilly. DR-positive maternal engrafted $T$ cells in a severe combined immunodeficiency patient without graft-versushost disease. Transplantation. 30:331-334.

34. Gill, T. J., III. 1977. Chimerism in humans. Transplant. Proc. 9:1423-1431.

35. Beer, A. E., and R. E. Billingham. 1973. Maternally acquired runt disease: immune lymphocytes from the maternal blood can traverse the placenta and cause runt disease in the progeny. Science (Wash. DC). 179:240242.
36. Hurtenbach, U., D. H. Sachs, and G. M. Shearer. 1981. Protection against graft-versus-host-associated immunosuppression in Fl mice. I. Activation of Fl regulatory cells by host-specific anti-major histocompatibility complex antibodies. J. Exp. Med. 154:1922-1934.

37. Naiman, J. L., H. H. Punnett, H. W. Lischner, M. L. Destiné, and J. B. Arey. 1969. Possible graft-versus-host reaction after intrauterine transfusion for $\mathrm{Rh}$ erythroblastosis fetalis. N. Engl. J. Med. 281:697-701.

38. Parkman, R., D. Mosier, I. Umansky, W. Cochran, C. B. Carpenter, and F. S. Rosen. 1974. Graft-versushost disease after intrauterine and exchange transfusions for hemolytic disease of the newborn. N. Engl. J. Med. 290:359-363.

39. Grogan, T. M., D. D. Broughton, and W. F. Doyle. 1975. Graft-versus-host reaction (GVHR): a case report suggesting GVHR occurred as a result of maternofetal cell transfer. Arch. Pathol. 99:330-334.

40. Sasazuki, T., Y. Kohno, I. Iwamoto, and M. Tanimura 1978. Association between an HLA haplotype and low responsiveness to tetanus toxoid in man. Nature (Lond.). 272:359-361.

41. Schanfield, M. S., J. V. Wells, and H. H. Fudenberg. 1979. Immunoglobulin allotypes and response to tetanus toxoid in Papua, New Guinea. J. Immunogenet. (Oxf.). 6:311-315.

42. Sumaya, C. V., and R. S. Gibbs. 1979. Immunization of pregnant women with influenza $A / N e w$ Jersey/76 virus vaccine: reactogenicity and immunogenicity in mother and infant. J. Infect. Dis. 140:141-146.

43. Murray, D. L., D. T. Imagawa, D. M. Okada, and J. W. St Geme, Jr. 1979. Antibody response to monovalent A/ New Jersey/8/76 influenza vaccine in pregnant women. J. Clin. Microbiol. 10:184-187.

44. Kerman, R., and D. Segre. 1970. Anti-pneumococcal polysaccharide type III hemolytic plaques in mice: cellular response in immunity and immunologic paralysis. J. Immunol. 104:1262-1266. 